Gut, 1968, 9, 683-687

\title{
Insulin potentiation of the augmented histamine response
}

\author{
R. G. CHECKETTS, I. E. GILlespie, AND A. W. KAY \\ From University Departments of Surgery, Royal Infirmary, \\ Sheffield, and Western Infirmary, Glasgow
}

The augmented histamine test has been widely accepted as an accurate and reproducible means of measuring the secretory capacity of the stomach.

It would appear that histamine requires the cooperation of cholinergic mechanisms to produce a full secretory response. Payne and Kay (1962) showed that the withdrawal of vagally released acetylcholine was mainly responsible for the large reduction in the augmented histamine response which had been observed after vagotomy; they were able to restore postvagotomy responses to almost preoperative levels by a simultaneous intravenous infusion of the stable cholinergic drug Mecholyl. The failure of Mecholyl to increase the augmented histamine response preoperatively may be a reflection of the already high level of cholinergic activity normally present in the human stomach. However, it has been suggested by several workers that the degree of vagal activity in duodenal ulcer patients may vary quite widely from individual to individual, and that in a small proportion of patients vagal activity may have a minor role in gastric acid secretion (Woodward, Harper, Tovee, and Dragstedt, 1949; Gillespie and Kay, 1961; Sircus and Small, 1964).

In the present study on duodenal ulcer patients attempts were made to potentiate the preoperative augmented histamine responses with the endogenous acetylcholine released at vagal nerve endings in the gastric mucosa by insulin hypoglycaemia.

\section{MATERIALS AND METHODS}

Sixty-seven male duodenal ulcer patients were studied, the diagnosis in all being confirmed at subsequent operation.

Each patient had two preoperative gastric secretion tests. On one day a standard augmented histamine test was performed after a 12-hour fast. A radioopaque nasogastric tube was passed under fluoroscopic control and the gastric secretion collected by continuous electric pump suction, with occasional interruption to ensure continuing patency of the tube. Four basal specimens were aspirated at 15-minute intervals after removal of the fasting juice. Then $50 \mathrm{mg}$ mepyramine maleate (Anthisan) and histamine acid phosphate, $0.04 \mathrm{mg} / \mathrm{kg}$ body weight, were given as a single combined intramuscular injection. Gastric aspiration was continued for a further three 15-minute periods, the response to the test being taken as the sum of the last two 15-minute outputs.

On a separate day insulin was given in addition to histamine. After collection of two 15-minute basal specimens, 10 units of soluble insulin was given intravenously. Fifteen minutes later $50 \mathrm{mg}$ mepyramine maleate and histamine acid phosphate $(0.04 \mathrm{mg} / \mathrm{kg}$ body weight) were given as a single combined intramuscular injection. Three further 15-minute aspirates of gastric secretion were again collected and the response to this combination of drugs was expressed as the acid output occurring in the second and third 15-minute periods after the histamine injection. The timing of the insulin in relation to the histamine was arranged so that the inhibitor effect of insulin on gastric secretion, which usually lasts about 30 minutes, would be likely to have passed by the time the histamine stimulation became maximal.

Postoperative insulin studies, using a dose of 20 units of soluble insulin, showed no increase in acid concentration greater than $10 \mathrm{~m}$-equiv/litre over basal levels at any time in a two-hour period after the insulin injection in 44 of the 67 patients, and in these a further augmented histamine test was performed about 10 days after operation.

The volume of each 15-minute specimen was measured. After filtration, acid concentration was calculated by titration against 0.1 normal sodium hydroxide with Töpfer's indicator. Acid outputs were derived by multiplication of volume and concentration values, and expressed as milliequivalents.

Potentiation was defined according to one of the criteria used by Gillespie and Grossman (1964) for urecholine and gastrin, and was considered to have occurred if the response to the combined agents exceeded the maximal response attainable by either agent alone.

\section{RESULTS}

The results in the 67 patients are presented in Table I. Statistical analysis using Student's $t$ test for paired values has shown that the mean response to the insulin plus histamine was significantly greater than the mean response to the augmented histamine 
TABLE I

EFFECTS OF INSULIN AND OF SURGICAL VAGOTOMY ON THE AUGMENTED HISTAMINE RESPONSE ${ }^{1}$

Patient No. Before Vagotomy

\begin{tabular}{ll} 
After Vagotomy & \\
\hline Augmented & $\begin{array}{l}\text { Percentage } \\
\text { Reduction by } \\
\text { Vagotomy }\end{array}$
\end{tabular}

Augmented Insulin + Percentage Change

Histamine Response

Augmented

by Insulin

Vagotomy

\begin{tabular}{|c|c|c|c|c|c|}
\hline 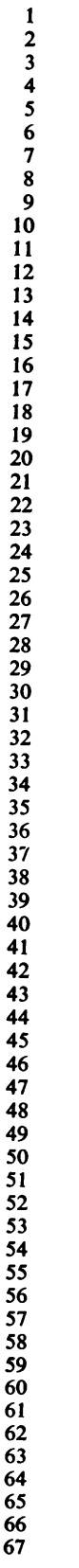 & 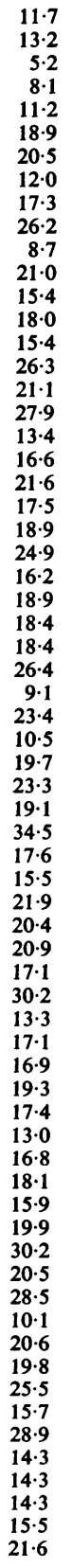 & 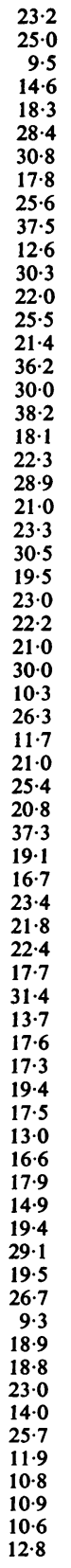 & 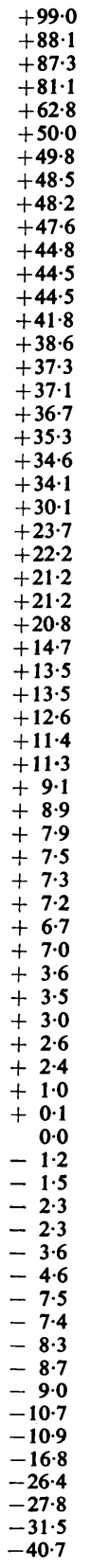 & $\begin{array}{r}0.0 \\
8.1 \\
\\
7.9 \\
3.9 \\
10.1 \\
\\
8.5 \\
3.6 \\
0.0 \\
\\
8.4 \\
5.7 \\
8.4 \\
0.5 \\
6.3 \\
5.0 \\
0.0 \\
0.5 \\
0.8 \\
\\
4.4\end{array}$ & $\begin{array}{r}100 \cdot 0 \\
65 \cdot 4 \\
\\
65 \cdot 8 \\
79 \cdot 5 \\
70 \cdot 7 \\
\\
45 \cdot 2 \\
83 \cdot 5 \\
96 \cdot 4 \\
\\
50 \cdot 5 \\
81 \cdot 0 \\
36 \cdot 7 \\
97 \cdot 2 \\
74 \cdot 5 \\
74 \cdot 0 \\
100 \cdot 0 \\
95 \cdot 6 \\
95 \cdot 4 \\
\\
72 \cdot 0\end{array}$ \\
\hline Mean & $18 \cdot 5$ & $21 \cdot 2$ & $+17 \cdot 7$ & $5 \cdot 6$ & $67 \cdot 7 \%$ \\
\hline
\end{tabular}

Pre-operative means for 44 patients in whom post-operative augmented histamine responses were obtained. $18 \cdot 3$

$21 \cdot 5$

$+20 \cdot 8$

${ }^{2}$ Values are expressed in $\mathrm{m}$-equiv $\mathrm{hcl} / \frac{1}{2}$ hour.

Percentage changes were calculated by taking preoperative augmented histamine response as $100 \%$. 
test alone $(\mathrm{t}=5.052 ; \mathrm{P}<0.001)$. The arithmetic mean of the percentage difference between each pair of test responses showed an overall increase in acid response of $17.7 \%$ when insulin was given in addition to the histamine. In 48 of the 67 patients the response to the insulin plus histamine was greater than to histamine alone and in 18 patients the response to the combined drugs was less than to histamine alone; in one patient the two responses were identical.

The average variation in duplicate augmented histamine tests in the same individual was recorded as $5 \%$ by Kay (1953) and $9 \%$ by Sircus (1960). Marks (1961) observed a similar degree of reproducibility, but expressed it as a coefficient of variation of $9.7 \%$. Although repeated augmented histamine tests were not performed on each of the patients in the present series, it would seem reasonable to accept a variation of up to $10 \%$ as being within clinical experimental error range. The acid response to the combined administration of insulin and histamine exceeded the response to histamine alone by more than $10 \%$ in 32 patients, ie, in $47.8 \%$ of the series. The largest increase in acid output from the combined drugs was $99 \%$ over the augmented histamine response. It seems likely that potentiation between the insulin and histamine had occurred in these 32 patients, according to the already mentioned criterion.

The responses to the two tests were within $10 \%$ of each other in 28 patients $(41.8 \%$ of the series), and in these potentiation clearly had not occurred. In seven patients $(10.4 \%$ of the series), the response to the insulin plus histamine was less than that to the histamine alone by more than $10 \%$, and it seems that the histamine response may have been inhibited by insulin.

The possibility of a relationship between the absolute value for the augmented histamine response and the change in it exerted by the insulin was explored by calculating the correlation coefficient for these two sets of values. The low correlation coefficient $(r=0.1232 ; t=0.998 ; \mathrm{P}<0.2)$ clearly shows that the level of the preoperative histamine response by itself is not necessarily related to the ease of achieving augmentation or inhibition by the added insulin.

A study of the acid responses in the 44 patients for whom postvagotomy augmented histamine responses were available revealed that there was, however, a relationship between the preoperative effect of the insulin on the histamine response, and the reduction in the augmented histamine response after vagotomy. A statistically significant negative correlation was obtained for the absolute change produced in the augmented histamine response by 6 insulin, and the absolute difference between pre- and postoperative augmented histamine responses. In other words, the greater the increase in the histamine response as a result of the added insulin, the smaller the reduction in histamine response after vagal section, and vice versa.

This relationship is more clearly demonstrated by subdividing the 44 patients into three groups, namely, (1) those in whom the insulin appeared to produce potentiation, as already arbitrarily defined (21 patients); (2) those in whom insulin produced no significant alteration (21 patients); and (3) those in whom the insulin appeared to inhibit the augmented histamine response (two patients). The average reduction in the augmented histamine response after vagotomy was $59.8 \%$ in group $1,77.5 \%$ in group 2 , and the reductions for the two individuals in group 3 were $93.2 \%$ and $100 \%$ respectively. There were eight patients in group 1 with a postoperative reduction in the augmented histamine response of less than $50 \%$, whereas in group 2 there were only two such patients.

\section{DISCUSSION}

It is of interest that insulin had a variable effect on the augmented histamine response in different individuals of the group of duodenal ulcer patients studied. Increase in the histamine response, probably amounting to potentiation, occurred in almost $50 \%$ of the 67 patients studied preoperatively, while inhibition of the histamine response was seen in $10 \%$, and no significant effect in the remainder. There would seem to be no satisfactory means of clearly defining true potentiation in such a series of clinical measurements, with the attendant variations for experimental error, and other sources of imprecision. It therefore seems impossible to establish an absolute level of difference between a histamine test and an insulin plus histamine one, above which potentiation can be confidently assumed, and below which it can be excluded. However, the indirect evidence which supports the occurrence of true potentiation, at least in those patients displaying the largest increase in acid output from the combination of insulin and histamine, are (1) that the overall mean acid output to the combined drugs is statistically significantly greater than that to histamine alone, and (2) that the uppermost value for experimental error in augmented histamine responses from published literature is exceeded in half of the patients studied. One of the criteria used by Gillespie and Grossman (1964) was that potentiation was considered to have occurred if the response to the combined agents exceeded the maximal response attainable by either agent alone. It would appear 
that those patients in the present series with a more than $10 \%$ increase in the augmented histamine response as a result of the added insulin satisfied this criterion as far as exceeding the maximal response attainable by the histamine alone. However, it might be argued that the increased responses were not demonstrated to be significantly greater than the maximal attainable by insulin alone. Although acid dose response curves to insulin were not performed in these subjects, there is evidence from the work of Ross (1964), in a study of comparable patients, that the mean acid response to the dose of insulin used in the present study was $70 \%$ of the maximal histamine response. Furthermore, in a detailed study of the gastric secretory response to graded insulin doses in experimental animals, Davis, Brooks, and Robert (1956) showed that increase in insulin dosage to approximately five times that used in the present study failed to evoke significantly larger acid responses. We conclude, therefore, that it is most unlikely that the maximal acid response to insulin alone would exceed the maximal histamine response, and that a response to the combined insulin plus histamine exceeding the maximal histamine response alone would satisfy the criterion already outlined. This would appear to be the first time that potentiation of the augmented histamine response in the intact stomach has been demonstrated, although Nechelles, Motel, Kosse, and Neuwelt (1938) did show cholinergic potentiation of submaximal doses of histamine in man.

It is interesting to speculate on the possible reason for a wide range of effects of the insulin on the augmented histamine response in different patients. The finding that vagotomy reduces the augmented histamine response, and that the simultaneous administration of a stable cholinester can restore this response to preoperative levels (Payne and Kay 1962) indicates that the augmented histamine response of the intact stomach is to some extent dependent on cholinergic participation. The degree of cholinergic contribution may vary from one individual to another. The hypoglycaemia which follows intravenous insulin probably stimulates the medullary vagal nuclei via the hypothalamus, resulting in a release of acetylcholine at the peripheral vagal nerve endings. During fasting conditions, the release of acetylcholine in the gastric wall, in any individual, may depend upon the level of tonic activity in the vagi. When vagal activity is already high, further release of acetylcholine in response to insulin hypoglycaemia may be too small to affect the augmented histamine response. A further possibility is that additional acetylcholine released by the insulin stimulation may increase the local gastric acetylcholine concentration sufficiently to cause a dose reversal phenomenon, similar to that described by Gray and Ivy (1937) for exogenous cholinergic activity in the form of Mecholyl, and confirmed by Gillespie and Grossman (1964) for Urecholine. These theoretical explanations could account for the failure to produce potentiation of the preoperative augmented histamine response in $41.8 \%$ of the patients, and for the inhibition of the response which occurred in $10.4 \%$. Potentiation between insulin and the histamine would then reflect a lesser degree of tonic vagal activity, still permitting increased stimulation from added insulin-induced acetylcholine. Thus it is possible that the effect of the insulin on the augmented histamine response may be quantitatively related to the spontaneous vagal activity in an individual patient.

Support for this hypothesis is given by the significant negative correlation coefficient for the change in the histamine response caused by the insulin, compared with the reduction of the augmented histamine response after vagotomy in the 44 patients in whom both pre- and postoperative data were available. This indicates that vagotomy would probably result in a large reduction in the augmented histamine response when insulin had produced little or no potentiation preoperatively; conversely, if considerable potentiation of the histamine response is obtained with insulin it would be likely that vagotomy would achieve only a small reduction in the augmented histamine response.

The failure of Payne and Kay (1962) to increase the preoperative histamine response by the simultaneous administration of intravenous Mecholyl may be due to several factors. It is possible that all five patients studied may have come from the group in which cholinergic activity seems to be spontaneously operating at near maximal level; alternatively it may be that Mecholyl administered systemically did not reach the exact site of action of acetylcholine in the gastric wall, or that the unpleasant side-effects prevented the administration of adequate amounts. These difficulties were fully discussed by Payne and Kay, and it was suggested that confining the cholinergic activity locally to the gastric tissues might yield different results.

In dogs a greater degree of potentiation between cholinergic drugs and histamine than that demonstrated in the present human study has generally been reported (Nechelles et al, 1938; Uvnäs, 1942; Gillespie and Grossman, 1964). In addition, the maximal histamine response is not significantly reduced in dogs by vagotomy (Hood and Code, 1950; Andersson and Grossman, 1965, Heathcote, Daly, and Gillespie, 1965). These findings, together with other evidence, are generally interpreted as indicating a lower vagal tone in dogs than in man. 
In a few patients vagotomy may not significantly reduce the amount of acid secreted, as exemplified by the small reductions in augmented histamine response in a minority of the present series. If it can be conclusively shown that ulceration may recur after complete vagotomy, and that the incidence of this complication is highest in such patients with a small reduction in secretory response, the combined insulin-histamine procedure might offer an alternative means to the attempted 'medical vagotomy' by atropine and hexamethonium (Gillespie and Kay, 1961; Abernethy, 1967) in the selection of patients for surgical treatment of duodenal ulceration.

\section{SUMMARY}

The effect of insulin-induced hypoglycaemia on the augmented histamine response has been studied in 67 duodenal ulcer patients. Augmentation of response, probably amounting to potentiation, was observed in some patients, in others inhibition occurred, while in a third group no significant effect was noted. It is suggested that the effects of insulin on the histamine response may be related to background vagal activity. The range of effects noted suggest that in a small number of duodenal ulcer patients factors other than the vagi may be dominant in the stimulation of acid secretion.

\section{REFERENCES}

Abernethy, R. J. (1967). The clinical application of gastric acid secretion studies. Brit. J. Surg., 54, 859-862.
Andersson, S., and Grossman, M. I. (1965). Effect of vaģal denervation of pouches on gastric secretion in dogs with intact or resected antrums. Gastroenterology, 48, 449-462.

Davis, R. A., Brooks, F. P., and Robert, C. M., Jr (1965). Gastric secretory response to graded insulin hypoglycemia. Amer. J. Physiol., 208, 6-8.

Gillespie, I. E., and Grossman, M. I. (1964). Potentiation between urecholine and gastrin extract, and between urecholine and histamine in the stimulations of Heidenhain pouches. Gut, 5 , 71-76.

on the augmented histamine test in man. Brit. med. J., 1 , $1557-1560$.

Gray, J. S., and Ivy, A. C. (1937). Effects of mecholyl on gastric secretion. Amer. J. Physiol., 120, 705-711.

Heathcote, B. V., Daly, D. W., and Gillespie, I. E. (1965). Secretory responses before and after vagal denervation of a gastric pouch. Gastroenterology, 48, 463-471.

Hood, R. T., Jr, and Code, C. F. (1950). Some effects of vagotomy on gastric secretion as studied in dogs with gastric pouches. Surg. Forum, 2, 73-78.

Kay, A. W. (1953). Effect of large doses of histamine on gastric secretion of $\mathrm{HCl}$ : an augmented histamine test. Brit. med. J., 2, 77-80.

Marks, I. N. (1961). The augmented histamine test. Editorial. Gastroenterology, 41, 599-603.

Nechelles, H., Motel, W. G., Kosse, J., and Neuwelt, F. (1938). The effects of acetylcholine, acetylbetamethylcholine and prostigmine on the secretion of the stomach of man and dog. Amer. J. dig. Dis., 5, 224-231.

Payne, R. A., and Kay, A. W. (1962). The effect of vagotomy on the maximal acid secretory response to histamine in man. Clin. Sci., 22, 373-382.

Ross, B. (1964). Human gastric secretion in response to insulin before and after surgical vagotomy. M.D. Thesis, Sheffield University.

Sircus, W. (1960). The aetiology of peptic ulceration. In Peptic Ulceration: a Symposium for Surgeons, edited by C. Wells and J. Kyle, pp. 11-36. Livingstone, Edinburgh.

- , and Small, W. P. (1964). The problem of peptic ulcer. Scot. med. J., 9, 453-468.

Uvnäs, B. (1942). The part played by the pyloric region in the cephalic phase of gastric secretion. Acta physiol. scand., 4, suppl. 13.

Woodward, E. R., Harper, P. V., Jr, Tovee, E. B., and Dragstedt, L. R. (1949). Effect of vagotomy on gastric secretion in man and experimental animals. Arch. Surg., 59, 1191-1212. 